\title{
At the interface of inorganic chemistry and biology
}

$\mathrm{H}$ ighlighting this issue of PNAS is a Special Feature comprising 11 Perspectives and 37 research articles covering various aspects of bioinorganic chemistry, the branch of chemistry that is concerned particularly with the properties and roles of metal ions in biology.

The recognition of the presence of iron-containing compounds in blood can be traced back at least as far as the 19th century. However, it was not until about 50 years ago that the systematic application of inorganic chemistry to the elucidation of the roles of metals in biological systems began to receive serious and sustained attention, and the field of bioinorganic chemistry began to evolve into the vigorous branch of science that it constitutes today. This evolution started with the mutual recognition by biological and medical scientists and by inorganic chemists of the significant connections between the themes of their research. Initially, these scientists identified with their original disciplines and interacted primarily at occasional meetings or symposia. With time this interface grew into a distinctive field, bioinorganic chemistry, manifesting the typical characteristics of a new scientific discipline: its own conferences, journals, societies, and a community of scientists who identify with the field and its designation.

A revealing record of the evolution of the field of bioinorganic chemistry is provided by the sequence of programs of the Gordon Research Conferences on Metals in Biology (originally designated
Metals and Metal Binding in Biology) that have been held regularly since 1962 . These conferences brought together a broad array of scientists from the field of inorganic chemistry with those from various biological sciences. Several circumstances contributed to making these interactions particularly timely and fruitful. First, inorganic chemistry, notably transition metal coordination chemistry, was undergoing a renaissance during the 1950 s and early 1960 s. This renaissance was fueled by the important new insights derived from applications of ligand field theory and from detailed theoretical and experimental studies on the basic reaction mechanisms of metal complexes. Second, at the same time, increasingly powerful structural, spectroscopic, and kinetic tools were exposing metal-containing biological systems to more detailed study and elucidation at the molecular level.

The programs of the early Gordon conferences in this series only hinted at the powerful links between inorganic chemists and biologists that were to evolve over the next few decades. Although both constituencies were well represented from the outset, their early contributions tended to focus on their own disciplines with only limited substantive overlap. During the ensuing period, and in considerable measure as a result of the communication and collaborations fostered by these and similar conferences, the community of scientists who constitute the "metals in biology" family today has become much more closely integrated, to the point that dis- ciplinary origins and affiliations have tended to merge and are no longer readily identifiable.

The collection of papers that makes up this special feature, so admirably summarized in the Perspective by Harry Gray, serves as an eloquent testimonial to how far this process has come. Consistent with the pattern of research activity in the field, most of the papers originate in academic chemistry departments. At the same time, at least half of them are coauthored with scientists in one of the departments of biological science or a medical school. It is hard to imagine a more appropriate theme for highlighting in a multidisciplinary journal such as PNAS.

The special feature on bioinorganic chemistry is one of a series of such collections of papers that PNAS has published in recent years, comprising solicited Perspectives and research articles focussed on specific cutting-edge multidisciplinary topics. Themes of previous features have included: Astrobiology; Evolutionary Developmental Biology; Rapid Climate Change; Social and Behavioral Sciences; and, most recently, Supramolecular Chemistry and SelfAssembly. One objective of these special features is to advance the journal's ongoing initiative to expand its coverage beyond its recent focus on biology. PNAS continues to encourage and welcome contributions of research articles in all areas of the natural and social sciences and mathematics.

Jack Halpern
Kenneth N. Raymond 